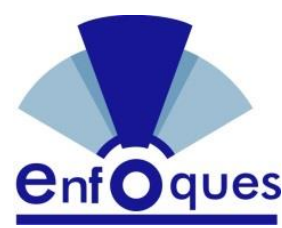

\section{LA GERENCIA SOCIAL UNIVERSITARIA EN VENEZUELA}

\author{
THE UNIVERSITY SOCIAL MANAGEMENT IN VENEZUELA
}

\section{Yarelis Verónica Quintero}

\begin{abstract}
Resumen
El artículo tiene como propósito, construir los elementos teóricos necesarios, que sustente lo constitutivo de la Gerencia Social Universitaria en Venezuela, desde la configuración de la UNEFA, la UNESR y la UBV. Con respecto a la metodología está enmarcada en el paradigma cualitativo, su enfoque es el interaccionismo simbólico. Los actores sociales son los docentes, empleados y estudiantes de las tres universidades tomadas como unidades de estudios y los cuales fungieron como informantes claves. La técnica de recolección de información fue la observación participante y la entrevista a profundidad. Para el análisis e interpretación de la información se utilizó la matriz GLATER, la cual sus autoras González y Hernández (2000) lo definen "como una matriz y un instrumento para evaluar las categorías provenientes de su investigación o de otros autores" (p.20). Para establecer la confiabilidad y valides se aplicó la triangulación. En la investigación sobresale la siguiente reflexión; el individuo que se desempeñe como gerente universitario le corresponderá tomar como marco de referencia los postulados de la gerencia social, toda vez que la misma es una gerencia compartida, solidaria, transparente, ajena a intereses sectoriales o corporativos; es una gerencia sensible e inteligente orientada al bien común, que desarrolla habilidades para la concertación, los consensos y los acuerdos.
\end{abstract}

Palabras clave: Gerencia social; gerencia universitaria; corpus axiológico

\section{Abstract}

The purpose of the article is to construct the necessary theoretical elements that support the constitutive of the University Social Management in Venezuela, from the configuration of UNEFA, UNESR and UBV. With respect to the methodology is framed in the qualitative paradigm, its focus is the symbolic interaccionalismo. The social actors are the teachers, employees and students of the three universities taken as units of studies and which served as key informants. The technique of data collection was participant observation and in-depth interview. For the analysis and interpretation of the information, the GLATER matrix was used, which its authors González and Hernández (2000) defined as "a matrix and an instrument to evaluate the categories coming from their research or from other authors" (p.20). To establish the reliability and validity, triangulation was applied. In the investigation the following reflection stands out; the individual who performs as a university manager will be responsible for taking as a frame of reference the postulates of social management, since it is a shared, solidary, transparent management, outside of sectoral or corporate interests; is a sensible and intelligent management oriented to the common good, that develops skills for the agreement, the consensuses and the agreements.

Key words: Social management; university; management and axiological corpus

Artículo recibido octubre 2016 | Arbitrado en noviembre 2016 | Publicado en enero de 2017
La Gerencia Social Universitaria en Venezuela Yarelis Verónica Quintero

No. 1 | Volumen 1 | Enero - Marzo 2017 http://doi.org/10.33996/revistaenfoques.v1i1.6 editor@revistaenfoques.org

ISSN: 2616 - 8219

\section{Yarelis Verónica Quintero}

yarelisquintero@gmail.com

Universidad Nacional

Experimental Simón

Rodríguez, Venezuela 


\section{(20) \\ INTRODUCCIÓN}

En el ámbito de la administración han surgido constantemente teorías que tratan de explicar a la organización y sus procesos, teniendo invariablemente como objetivo, la máxima eficiencia de las mismas.

Al respecto, la administración científica, intentó aplicar el método científico a los problemas de la administración, con el fin de maximizar la producción industrial. La teoría clásica, concibe la organización como una estructura al igual que la administración científica, su objetivo es la búsqueda de la eficiencia de las organizaciones a través de las mejoras de sus procesos, la teoría neoclásica le asigna gran relevancia a los aspectos prácticos de la administración. Incorporándoles conceptos clásicos de: estructura, autoridad, responsabilidad y departamentalización.

Así como han surgido teorías administrativas, en esa misma medida han emergido modelos gerenciales con idéntico propósito; maximizar la eficiencia de las organizaciones, en este caso enfocándose a las personas que la dirigen (el gerente). Entre estos modelos, se pueden mencionar: planeación estratégica, calidad total, kaizen - mejoramiento continuo, justo a tiempo just in time - (j. i. t.), benchmarking, reingeniería, desarrollo a escala humana y empoderamiento - "empowerment".

Todos estos modelos tienen un elemento en común, tienden a visualizar al gerente como un líder dentro de las organizaciones, es decir, la persona que dirige, planifica, organiza, controla y evalúa los procesos administrativos y de producción. Cuando en una sola persona recaen todas estas responsabilidades, genera que el gerente se torne autocrático, inflexible e incapaz de escuchar, posibilitando que surja en el personal la incertidumbre.

Al respecto, Dichter (1988) señala que el término "gerente es un eufemismo para designar el acto de guiar a los demás, lograr que las cosas se hagan, dar y ejecutar órdenes". (p.3).

En se mismo orden de ideas, las revistas "Debates", Instituto de Estudios Superiores de Administración (IESA 1999) y Calidad Empresarial (2000), han publicado una serie de artículos referidos: a la gerencia y sus modas, características del líder del 2000, los cuales entre otros aspectos manifiestan, que los gerentes deben afianzar sus conocimientos, tener visión de futuro, amplitud de juicio, mentalidad al servicio del cliente.

Estas publicaciones ratifican que la tarea de los gerentes, es vincular estrechamente sus metas de desempeño individual con las metas de la empresa, garantizar la productividad de las mismas y su permanencia en el tiempo, dejando a un lado lo humano de la organización, es decir, que no se interesan en desarrollar las relaciones humanas, se enfocan prioritariamente a la consecución de las metas.

Las organizaciones que así actúan se han quedado ancladas en los postulados de la administración científica de Taylor (1911). Organizaciones de este tipo podrán ser eficiente pero nunca humanas, olvidando que el talento humano es el que le da vida a éstas, las cuales existirán en la medida que haya personas capaces de comunicarse, que estén dispuestas a actuar conjuntamente para obtener un objetivo común.

La investigadora presume, que las casas de estudios universitarios se encuentran inmersas en el planteamiento antes referido, en cuanto en el interior de estas organizaciones se puede observar el 
desmejoramiento en las relaciones interpersonales, grupos de personas trabajando aisladamente, sin comunicación alguna, autoridades que solo imparten ordenes sin sopesar el impacto que las mismas pueden generar en el personal, entre otros. Situación que afecta negativamente lo axiológico, condiciones que desfavorecen sus actividades principales, como es lo académico administrativo.

Es por ello, que en la actualidad, cada vez más adquiere mayor valor la necesidad de un desempeño ético en la gestión de las universidades que colabore con una praxis gerencial oportuna en los disímiles intereses que coexisten en las mismas, siendo oportuno plantear una reforma universitaria que dé respuestas a las nuevas realidades a la que está expuesto el sector universitario.

En tal sentido, la autora discurre que la reforma universitaria, tendría por objetivo central, responder positivamente a las demandas sociales para la democratización substancial de las casas de estudios públicas haciendo realidad la construcción colectiva de saberes. Ello sentaría las bases para mitigar una historia de exclusión de grupos sociales, de la cual ha sido protagonista dichas instituciones durante un largo tiempo.

Así mismo, las universidades venezolanas del sector público, se plantean un reto, ya que las mismas presentan una gerencia tradicional, entendiendo esta como el proceso de planificación, organización, dirección, coordinación y control, dejando de lado elementos como la comunicación, el liderazgo, la toma de decisiones y el clima organizacional, quedándose en un letargo administrativo y por ende, no han sido capaces de afrontar los cambios del contexto actual, situación que ha generado un manejo inadecuado de los recursos asignados y el deterioro del desempeño de dichas instituciones, en detrimento de todos los sectores que allí confluyen, dentro de esta perspectiva, en la Universidad de los Andes (ULA) en boletín publicado, Papeles para el Cambio por los autores Malpica. R y Rossell. $R$ (2010), plantean lo engorroso de los procesos de gestión universitaria, exponiendo que: Es compleja y, a pesar de que existen muchos documentos en donde se expresan objetivos, el sistema jerárquico, los procesos, las políticas, entre otros elementos, es notoria la imprecisión, ambigüedad y poca capacidad operativa de los mismos. La ausencia de objetivos claros afecta las decisiones, sus procesos, la evaluación de sus logros, la definición de la calidad de sus procesos y resultados (p. 35).

De lo antes planteado, se desprende que las universidades públicas, presentan complejidad en su gestión, muchas de estas generadas por improvisación, ausencia de cohesión de equipos y desconocimiento de los procesos. Monagas (2003), considera que se debe concebir la gerencia universitaria "como el manejo de los procesos administrativos-académicos cuyo fin es la búsqueda del cumplimiento de los objetivos institucionales". (p.10).

En este mismo orden de ideas, López (1998) (citado por Monagas 2003) agrega que: ... las universidades más que gerentes, estas tienen jefes, generalmente sin formación gerencial alguna, quienes llegan a esos cargos por razones políticas o por decisiones grupalistas. Ciertamente es así, y por ello es necesario revertir tal situación si se pretende tener una verdadera gerencia universitaria que posibilite el desarrollo y éxito de la institución (p. 21).

Es por ello, que un gerente universitario en términos operacionales requiere de habilidades propias de la gerencia social, la cual propone un modelo 
participativo-incluyente de fondo y no meramente formal; no se trata de la participación del "buzón de sugerencias", sino de la participación efectiva del necesitado; pasar del discurso magistral y demagógico, de lo que se debe hacer, a hacerlo con el otro; pasar de la oratoria a la "escuchatoria"; (que no es más que el escuchar con atención, para captar con la mayor claridad posible el fondo del mensaje, buscando sus intenciones y motivaciones y no solo conformarse con la ideas superficiales del mismo) generar confianza, credibilidad y estar sensibilizado para producir resultados que impacten significativamente en el bien común.

Licha (1999). Expresa que: La gerencia social tiene su propia especificidad y autonomía, que la hace distinta de la gerencia privada, movida por otra lógica y propósitos, y también de la gerencia burocrática tradicional, rígida, centralizada, poco transparente y poca participativa. Su singularidad estaría dada por las características propias de las políticas sociales en sociedades profundamente desiguales, instituciones débiles $y$ democracias precarias. (p. 12)

Bajo esta perspectiva, el Estado Venezolano, para profundizar el cambio cultural, social, y educativo, que el país transita desde 1999 y afianzar la praxis axiológica en las universidades, se plantea la aplicación del modelo de la gerencia social, con la creación el 18 de julio de 2003, mediante decreto Presidencial № 2.517, la Universidad Bolivariana de Venezuela (UBV) la cual surge como una institución de educación universitaria, alternativa al sistema educativo tradicional y transfigurada en una nueva forma de gerenciar, desde lo social y lo endógeno, para la masificación de la educación.
La Universidad estará abierta no sólo al pensamiento universal y a la sociedad, sino también al cambio de nuestros esquemas mentales tradicionales, que den paso a la imaginación creadora, al entendimiento del país comprendiendo su historia y su inserción en el mundo, al ejercicio cabal y libre de la democracia participativa y la discusión permanente del concepto de Universidad. Todo esto implica la construcción de una comunidad universitaria, la búsqueda de la calidad, la pertinencia, la equidad y la convivencia democrática, como factores fundamentales para su desarrollo institucional.

Así mismo, el Estado se apoyó en otras instituciones tales como; la Universidad Nacional Experimental de las Fuerzas Armadas (UNEFA) antiguo Instituto Universitario Politécnico de las Fuerzas Armadas Nacionales (IUPFAN), Fundada el 16 de agosto de 1973, por decreto de Rafael Caldera, en sus 25 años de funcionamiento, esta institución educativa fue una alternativa para la Educación Superior de los miembros de las Fuerzas Armadas Nacionales y de la comunidad en general. El 26 de abril de 1999, y esta vez por decreto del presidente Hugo Rafael Chávez Frías, pasa a ser UNEFA.

Por último, se encuentra la Universidad Nacional Experimental Simón Rodríguez (UNESR), la cual se suscribe a los principios fundamentales del hombre, la educación liberadora, la andragogía y la herencia de progreso y pensamiento de nuestro epónimo Simón Rodríguez. Igualmente, define su papel académico, con base en la capacidad transformadora y la conciencia social, las cuales marcan el punto de partida para formar profesionales críticos, conocedores de sus deberes y derechos, con sentido ético, sensibilidad humana y visión colectiva. 
Así mismo, está orientada a consolidar una universidad capaz de formar los promotores y conductores de la nueva realidad nacional, basada en la universalización de la educación y en la colectivización del saber, colocando el mismo al servicio de las comunidades, con la firme convicción de estrechar la brecha entre las casas de estudios y las personas que estuvieron excluidas por décadas, es decir, como proceso homólogo a la inserción colectiva de ciudadanos y ciudadanas a los ambientes de construcción de saberes, con el fin, de hacer extensiva la universidad y sus procesos formativos a la comunidad en general.

Con esta finalidad, las dos primeras universidades mencionadas nacen con un enfoque, humanista, promoviendo valores de equidad, igualdad, justicia, participación entre otros, entendido esto como un proceso de evolución de trascendencia social, el cual ha tenido un impacto significativo hacia los estudiantes que hacen vida universitaria, dándoles la oportunidad de incorporarse al quehacer universitario a través de las vocerías estudiantiles, entre otros.

Sin embargo, para que se pueda visualizar una transformación universitaria equilibrada, se ha de dar participación igualitaria a todos los que hacen vida en las casas de estudio, entiéndase éstos, como Obreros, Empleados, Estudiantes y Docentes, convocándolos a la concertación, el consenso y acuerdos en materia académica administrativas, con la intencionalidad de cambiar la forma de gerenciar las instituciones universitarias, haciendo realidad de esta manera lo establecido en la Constitución de la República Bolivariana de Venezuela (1999), cuando expresa en su preámbulo, “....establecer una sociedad democrática, participativa y protagónica, multiétnica y pluricultural..." (p. 1)

Ante estos planteamientos y de acuerdo con la realidad esbozada en el abordaje del objeto de investigación, la problemática surge a partir del no cumplimiento con lo establecido en la Ley de Universidades (1970) en la cual se plasma, que las autoridades de las universidades nacionales se deben escoger vía electoral, tres meses antes de vencer su periodo de cuatro años como lo refiere el artículo 30 de dicha ley. Sin embargo, dicho documento legal no está cónsono con los nuevos tiempos, es decir, que no garantizan la participación protagónica de todos los actores sociales que confluyen en las universidades públicas, ya que esta limita la participación en la elecciones a los facilitadores (asistentes, agregados, asociados, titulares y jubilados) desconociendo a los instructores, en el caso de los estudiantes no puede votar el colectivo, se escogerá los representantes que será igual al $25 \%$ del claustro universitario, esto enmarcado en los numerales 1 y 2 del referido artículo.

De lo que se puede argüir, que la misma, deja fuera a los obreros y administrativos, vulnerando con ello lo establecido en el artículo 15 numeral 2 de la Ley Orgánica de Educación, el cual expresa que uno de los fines de la educación es "Desarrollar una nueva cultura política fundamentada en la participación protagónica y el fortalecimiento del Poder Popular, en la democratización del saber y en la promoción de la escuela como espacio de formación de ciudadanía y de participación comunitaria, para la reconstrucción del espíritu público en los nuevos republicanos $y$ en las nuevas republicanas con profunda conciencia del 
deber social". (Subrayado de la investigadora).

Es por ello, que impulsar la participación protagónica de todos los sectores que hacen vida dentro de las universidades públicas seleccionadas, redefiniría la gerencia social universitaria, en sus relaciones con los actores que le dan vida a estas casas de estudios. Se modificaría con ello patrones de conducta, creencias, visiones y prácticas democráticas, identificadas con la representatividad, y se trasciende a la ejecución de acciones que, en lo concreto, se enmarcan en la democracia participativa y protagónica, tal y como se establece en la constitución nacional.

En atención a lo antes planteado, el tema objeto de estudio está enfocado a develar la necesidad de las personas que funjan como gerente universitario para conectarse con su ser y de allí, pueda emerger a una gerencia social, sustentada en valores de equidad, justicia, igualdad, respeto, comunicación, liderazgo y toma de decisiones, entre otros, que coadyuven a consolidar la universidad pública actual y trasciendan en el tiempo como "anticipadora" de tendencias generadoras de conocimientos y formadora de los futuros profesionales que demande la sociedad.

En tal sentido, metodológicamente para abordar dicho trabajo se realizó un arqueo bibliográfico para seleccionar las teorías con las que se desarrolló el tema. El paradigma escogido fue el cualitativo, el enfoque el interaccionismo simbólico, el cual adjudica primordial importancia a los significados sociales que las personas le otorgan a los objetos que les rodean mediante símbolos (Blumer, 1969). Se utilizó la entrevista a profundidad, así mismo los actores sociales son; los docentes, empleados y estudiantes de las tres universidades tomadas como objeto de estudio y los cuales fungieron como informantes claves.

En este mismo orden de ideas, la técnica de recolección de información utilizada fue la observación y la entrevista a profundidad. Los instrumentos de recolección de información fueron el guion de entrevista y el diario de campo. Para sistematizar la información se utilizó la matriz GLATER, definida por sus autoras, González y Hernández, (2000) “como una matriz y un instrumento para evaluar las categorías provenientes de su investigación o de otros autores" (p. 20).

Así mismo, se empleó la triangulación, la cual es una estrategia de control para avalar la confiabilidad entre los resultados de cualquier investigación. (Donolo, 2009:53), los resultados que han sido objeto de estrategias de triangulación pueden mostrar más fuerza en su interpretación y construcción que otros que han estado sometidos a un único método.

Denzin (1970) plantea que: El uso de tres o más perspectivas o diferentes observadores o varias fuentes de datos cualitativos/cuantitativos o estadísticos distintos, es llamado el método de triangulación: estudios, perspectivas, investigadores, datos y estadísticos. Tres al menos, es una garantía de fiabilidad o robustez y asimismo sirve para reducir las replicaciones y también suprimir la incertidumbre de un solo método (p.25).

\section{Plataforma Teórica - Conceptual}

\section{Teoría Gerencial}

La gerencia es el cargo que ocupa una persona dentro de una organización, y él cual debe cumplir múltiples funciones, entre ellas, representar a la sociedad frente a terceros y coordinar todos los recursos a 
través del proceso de planeamiento, organización dirección y control a fin de lograr objetivos establecidos.

Sisk y Sverdlik (1976) expresa que el término gerencia: Es difícil de definir: significa cosas diferentes para personas diferentes. Algunos lo identifican con funciones realizadas por empresarios, gerentes o supervisores, otros lo refieren a un grupo particular de personas. Para los trabajadores; gerencia es sinónimo del ejercicio de autoridad sobre sus vidas de trabajo... (p.136)

De allí que, en muchos casos la gerencia cumple diversas funciones porque la persona que desempeña el rol de gerenciar tiene que desenvolverse como administrador, supervisor, delegado, entre otras. Otros autores como. Crosby (1988) define a la gerencia como "el arte de hacer que las cosas ocurran". (p. 120).

Por su parte Krygier (1988) la define como un cuerpo de conocimientos aplicables a la dirección efectiva de una organización (p. 215).

A lo plateado se puede argüir, que la gerencia, hoy requiere de mucha información oportuna y veraz; de sagacidad y astucia para afrontar las dificultades; de creatividad e ingenio para formular las soluciones; de contacto permanente con el mundo exterior a la organización.

Es importante destacar que los cambios ocurridos en el mundo no sólo han afectado a las industrias privadas sino a todos los actores sociales, las apreciaciones que se examinaron sobre el tema gerencial hay que hacerlas extensivas a todos los agentes que participan en la organización. El caso es que ahora todos esos principios se tienen que aplicar también en las empresas de servicio y en las del Estado y aún en la administración central del mismo.

\section{Gerente}

Dichter (1988) señala que el término "gerente es un eufemismo para designar el acto de guiar a los demás, lograr que las cosas se hagan, dar y ejecutar órdenes". (p.17)

De lo antes planteado, se puede inferir, que el gerente es el individuo que dirige una empresa por cuenta y encargo del empresario. En éste se delega la labor de cuidar, supervisar, controlar, planificar, las actividades de las personas que bajo su mando están. Para ello, el mismo debe contar con habilidades o destrezas que le permita ejecutar su función.

\section{Gerencia Social}

Rojas (2008) "La aparición del concepto de gerencia social es un ejemplo claro de que, más que acercarnos a una sociedad sin gobierno, nos enfrentamos a una nueva forma de gobernar lo social”. (p. 2)

En el nuevo contexto socio-político y conceptual por el que viene atravesando el país, la gerencia social adquiere connotación diferente a la que tiene para las políticas tradicionales.

A lo que Guendel (2001) considera que tiene tres premisas. En primer término, se agregan nuevos temas que nunca había sido tratados por los planificadores y comunicadores sociales. Se requieren, por consiguiente, nuevas aproximaciones a lo social, un ámbito que se ha enriquecido y se ha ampliado significativamente en virtud del "redescubrimiento" de las identidades, nuevos enfoques de atención y precisiones discursivas que establezcan la interfase entre el discurso y la práctica social. En segundo término, se generan exigencias movilizadoras de nuevo cuño, que requiere un sujeto distinto: informado, reflexivo $\mathrm{y}$ 
activo, dispuesto a participar activamente en los procesos de configuración social. En tercer término, se exige un tipo de planificador social que asuma un rol de facilitador y conductor de procesos, ajustado a la perspectiva del Estado como medio y como organizador de un sistema de protección integral, que articule una amplia red de responsabilidad del cumplimiento de los derechos compartida por la comunidad, las organizaciones corporativas, la persona y el mismo Estado (p. 4).

Este nuevo enfoque de la gerencia social, apunta a construir una nueva cultura social e institucional que se exprese en prácticas sociales ajustadas a los aspectos de equidad, valores, justicia, igualdad $y$ en quehaceres institucionales dirigidos al fortalecimiento del proceso de construcción de los saberes para la transformación de la realidad social en función del conocimiento, que redefinan el desarrollo del país y coadyuve en articular las perspectivas gerenciales con las dimensiones de la ética y la moral institucional.

Kliksberg (1995) dice que, el verdadero desafío en materia de reforma del sector social es superar las limitaciones de su perfil actual, caracterizado por organizaciones aisladas poco flexibles, hipercentralizadas, hostiles a la participación real, alusivas de los problemas de lucha por el poder, renuentes a la concertación, de peso limitado en el aparato público, sin carreras gerenciales orgánicas, ni gerentes especializados en lo social. (p.7).

En tal sentido, se puede argüir que la gerencia social requiere ciertas formas muy particulares de acción dado las características del contexto actual venezolano y de los programas sociales del mismo.

Al respecto, Kliksberg (1997) anota que: La gerencia social eficiente tiene que ver con optimizar el rendimiento de los esfuerzos de los actores sociales en el enfrentamiento de los grandes déficits sociales de la región y el mejoramiento del funcionamiento y resultados de la inversión en capital humano y capital social (p.20)

Se necesita un enfoque de gerencia social que coadyuve a neutralizar los efectos sociales negativos del modelo neoliberal en las universidades públicas. Es decir, que se mitigue el autoritarismo de los gerentes que dirigen estas casas de estudio, que estén en concordancia con los nuevos tiempos, que aplique una gestión basada en la equidad, justicia, inclusión, liderazgo, comunicación y todos los valores constitucionales implantados en la carta magna por la que se rige el país de manera que estas propicien impactos reales en materia de desarrollo humano y produzcan los cambios o transformaciones que la nación está demandando.

En tal sentido, se debe gestar un concepto de "gerencia social", donde los factores determinantes de los objetivos surjan de las propias realidades existentes, en los campos institucionales, jurídicos, ideológicos, políticos, económicos, organizacionales, entre otros. Sólo de esta manera se podría, avanzar en materia de desarrollo humano. Cabe entonces preguntarse ¿Las universidades venezolanas funcionarían eficaz y eficientemente en la construcción y transformación del saber en pro de las necesidades del contexto actual en el país si el Hombre que la dirige, es decir, (el gerente universitario, actuara bajo la perspectiva de la gerencia social).

\section{Gerencia Universitaria}

Clemenza y Ferrer (1999) sugieren que: La universidad debe constituirse en un ámbito donde la labor educativa sea contextual, orientadora, formadora $y$ 
y creadora de conocimiento. Esta se enfrenta a grandes retos que la impulsa a transformar su estructura académica - administrativa para que su capacidad de respuesta sea segura y rápida, de tal manera que pueda adecuarse a las nuevas demandas del entorno interno y externo, quien exige mejoramiento continuo, eficiencia, eficacia y efectividad social. (p. 97)

Miguilena (1999) concibe a la universidad como un agente de transformación social, la misma y tiene el compromiso de formar el talento humano que necesita el país. Más sin embargo estos requerimientos deben ir de la mano, con un sistema educativo con un alto nivel de eficiencia, lo que se debe traducir en una gerencia efectiva. (p.42)

De acuerdo a lo establecido en la Ley de Universidades (1970) la gerencia es uno de los órganos unipersonales básicos de las universidades públicas.

Guijarro y Chávez (2006). Plantean que; las universidades deben adecuar sus esquemas gerenciales con la finalidad de alcanzar calidad, eficiencia, eficacia, pertinencia, equidad y producción de bienes sociales que le permitan establecer relaciones entre las asignaciones presupuestarias y el cumplimiento de sus funciones básicas (docencia, investigación, extensión) según los niveles que exige y demanda la sociedad. (p. 201).

En este orden de ideas, la universidad debe enfocarse en la administración y desarrolla de sus funciones vitales, antes mencionadas, las cuales deben reposar sobre una plataforma gerencial eficaz, eficiente $y$ racional en el proceso administrativo académico, lo cual garantice satisfacer las necesidades de la nación.

Picón citado por Muro (2003) enfatiza que: La gerencia universitaria será más eficiente en la medida en que interprete la misión de la universidad en su doble exigencia de afianzar los valores trascendentales del hombre y de contribuir a establecer y resolver sus problemas y necesidades, y proceda de acuerdo con su interpretación. (p. 160)

En consecuencia, la gerencia universitaria requiere de continuidad en el proceso administrativo - académico. En cuanto es el centro de las acciones para que la institución sea eficiente, y poder dar una imagen de excelencia y productividad. En este sentido, la gerencia debe hacerse de enfoques, modelos y paradigmas innovadores para rebatir la gerencia tradicional y formal, para adecuarse a los nuevos tiempos e inicie un camino para un nuevo liderazgo gerencial, o más bien una gerencia universitaria desde la perspectiva de la gerencia social que implica la participación de los empleados, obreros, facilitadores, estudiantes y comunidad, la modernización de los procesos, la innovación, la equidad, la calidad y la productividad en la transformación y construcción de la realidad social (ver figuras 1, 2, 34$)$. 


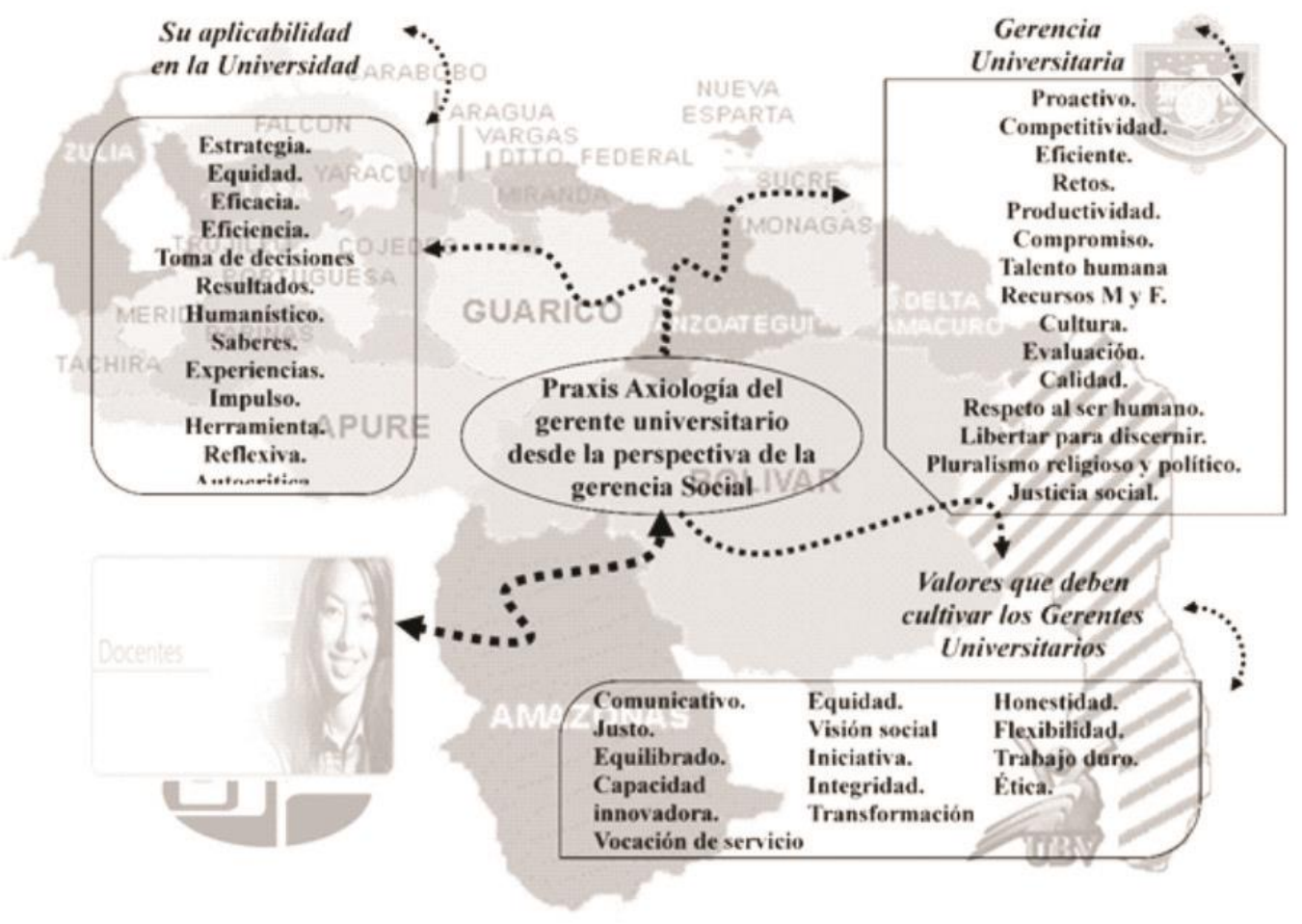

Figura 1. Estructura de hallazgos según informantes (Docentes o Facilitadores)

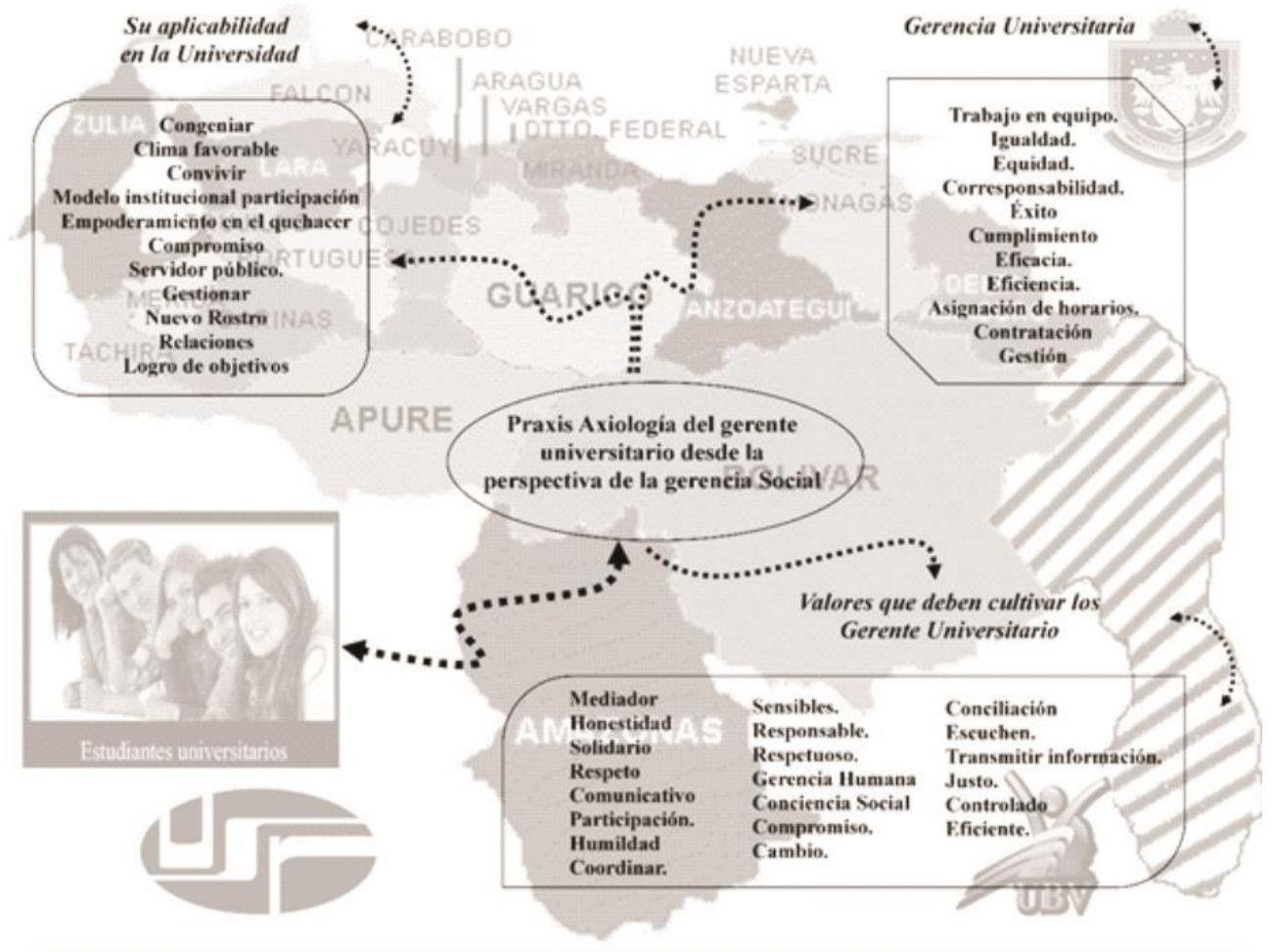

Figura 2. Estructura de Categorías según informantes (Estudiantes o Participantes). 


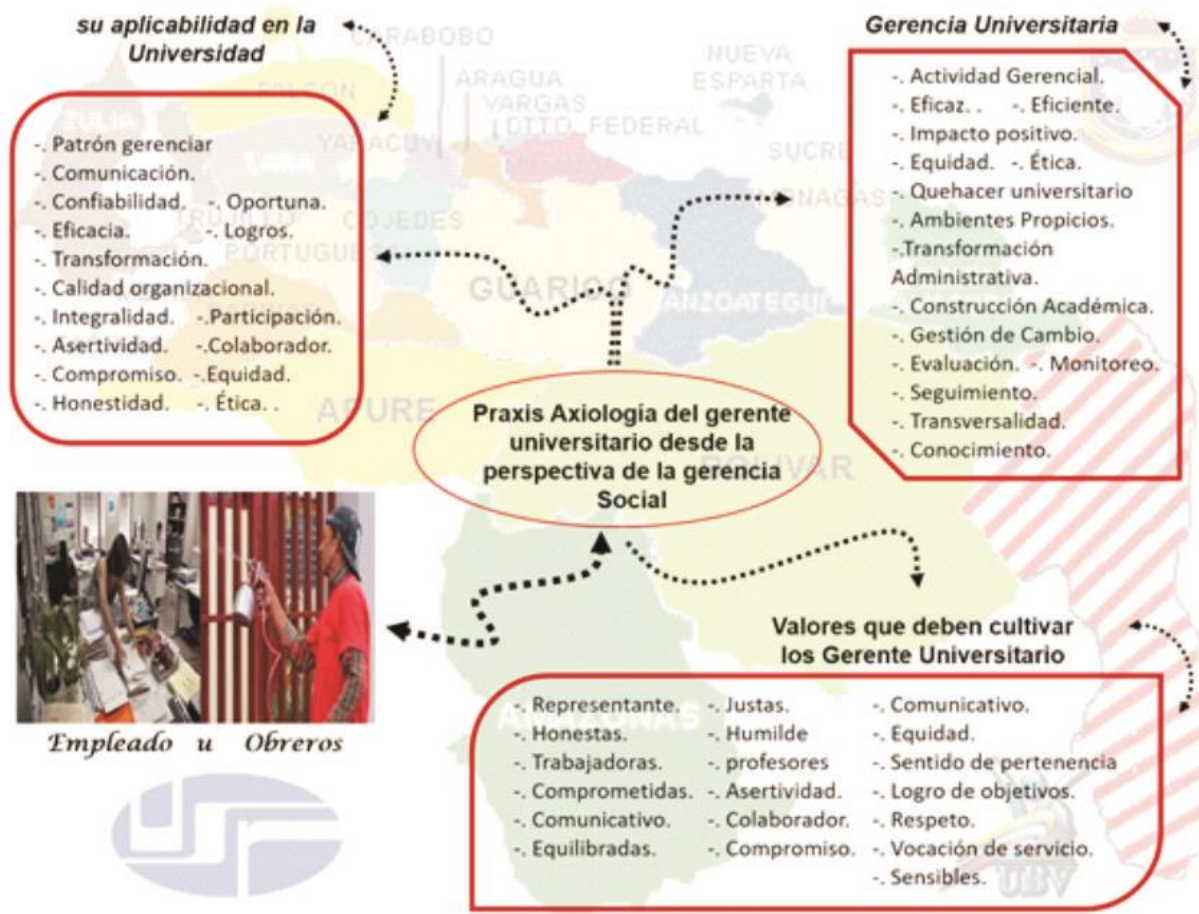

Figura 3. Estructura de Categorías según informantes (Empleados u Obrero).

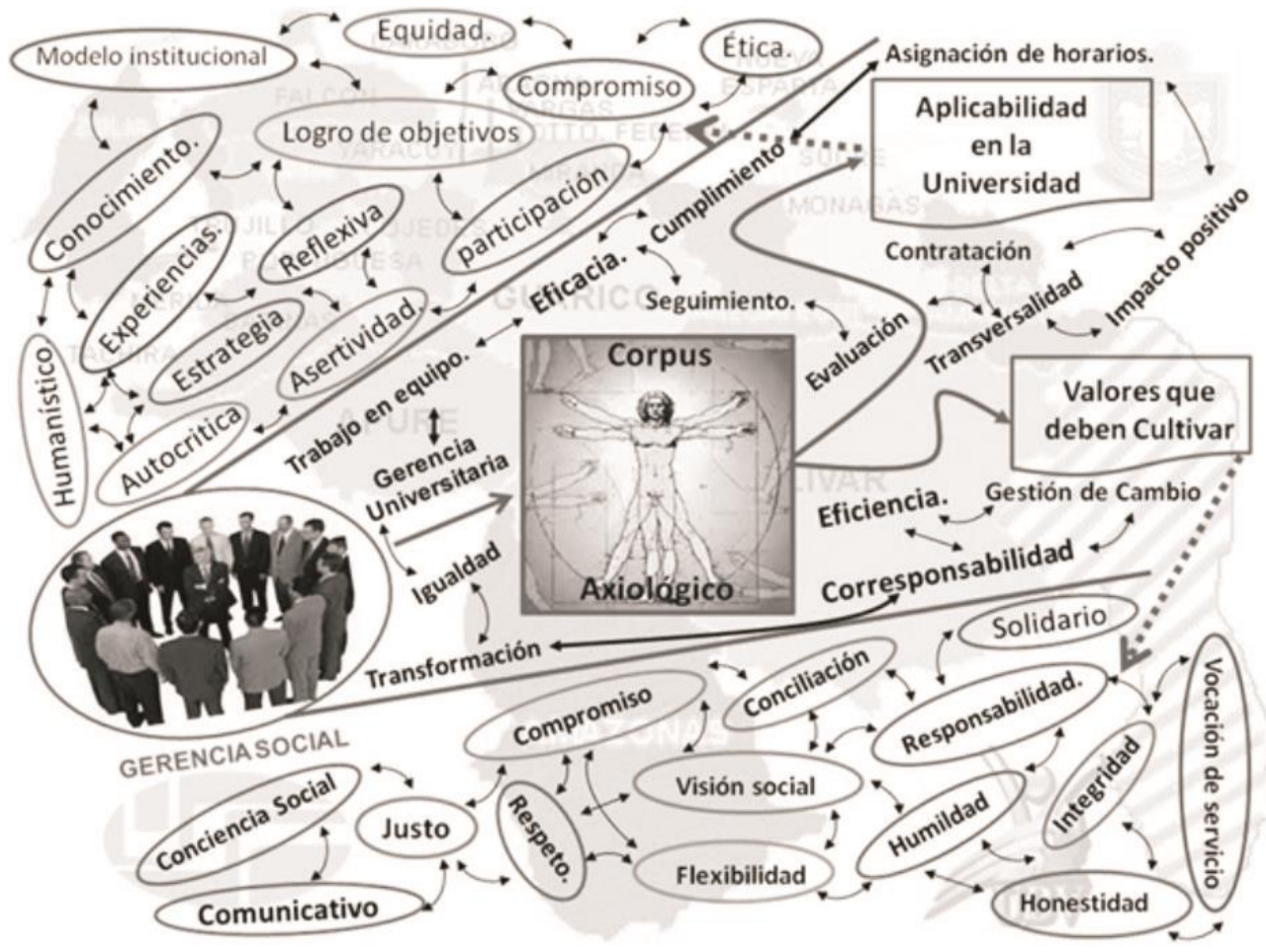

Figura 4. Estructura del Corpus Axiológico según los hallazgos del fenómeno. 


\section{Reflexionando sobre el fenómeno}

Los desafíos no se declaran, más bien se asumen con responsabilidad, disposición y compromiso de todos los actores sociales. Lo que demanda la transformación universitaria, es la convicción de que es el tiempo de abrir todos los espacios de reflexión, discusión y diálogo, con relación a lo que ha sido, es y puede ser el gerente universitario enmarcado en una praxis axiológica que caractericé la gerencia social en las universidades públicas venezolanas.

Por ende, es oportuno, plantear en este último espacio de reflexión que por demás acompaña el desarrollo del estudio en toda su esencia, lo siguiente:

Dentro de las conceptualizaciones manejadas en el desarrollo de la investigación la autora infiere que la gerencia social apuesta a la convivencia igualitaria, concatenando los intereses individuales y colectivos de los individuos sustentados en valores, adecuando la transformación de la praxis del gerente universitario, así propiciar el aprendizaje y la corresponsabilidad como elementos viables e indispensables, para generar competencias en el campo académico - administrativo de las instituciones de construcción de saberes.

Por lo tanto, el individuo que se desempeñe como gerente universitario le corresponderá tomar como marco de referencia los postulados de la gerencia social, toda vez que la misma es una gerencia compartida, solidaria, transparente, ajena a intereses sectoriales o corporativos; es una gerencia sensible e inteligente orientada al bien común, que desarrolla habilidades para la concertación, los consensos y los acuerdos.

De allí pues, que el gerente universitario que transite bajo los parámetros del enfoque social debe dimensionar herramientas como la gestión del cambio, la comunicación, la evaluación, el seguimiento y la sistematización de los objetivos planeado en lo académico - administrativo, para evitar generar intervenciones poco articuladas en las diversas actividades que se planteen en la institución. En virtud de que dichas herramientas le proporcionaran elementos de replicabilidad, sostenibilidad y medición del impacto, de la reflexión permanente acerca de cómo, en donde, con qué, y con quienes se pueden alcanzar las metas.

Sobre la base de las ideas del corpus axiológico que se esbozó para la gerencia social, resulta claro, que es el trazo colaborativo de un grupo de actores inmersos en el mundo universitario, con necesidades e inquietudes semejantes, emergiendo y conceptualizando un nutrido conjunto de valores reflejados en el momento V, En este sentido es pertinente resaltar los siguientes: en primer lugar la Humanidad: Es tener como valor central al ser humano y no cualquier otra cosa, es reconocer la diversidad, el potencial de cada individuo y asumirla como algo positivo, para la conformación de los equipos de trabajos, entender que todos tenemos necesidades individuales, luchar por ser más justo, equitativo y así brindar mayor oportunidad de desarrollo personal, en pro de la organización, y en segundo lugar la Humildad: Es la capacidad de un individuo de reconocer las potencialidades, habilidades y destreza de las personas y tratar de aprender de ellas, es propicio citar a Schütz (1995) el cual esgrime:

(...) $\mathrm{Mi}$ experiencia del mundo se justifica y corrige mediante la experiencia de los otros; esos otros con quienes me interrelacionan conocimientos comunes, tareas comunes y sufrimientos comunes. El mundo es interpretado como el posible 
campo de acción de todos nosotros: este es el primero y más primitivo principio de organización del conocimiento del mundo exterior, en general. Con posterioridad, discrimino entre cosas naturales (...) y, por otra parte, cosas sociales, comprensibles únicamente como productos de la actividad humana, mi propia actividad o la de otros (p.22).

La afirmación anterior, permite a los individuos reconocerse como ser humano con capacidad para producir, amar, jugar, gozar, relacionarse con el otro, como elemento dinámico. Permite reconocer sus potencialidades y las de los demás, como también ser capaz de actuar por iniciativa propia en la formulación, cogestión y autogestión de propuestas orientadas a la satisfacción de sus necesidades individuales y colectivas en pro del bien social.

En consecuencia, cobra vigencia los postulados de blúmer en función del Interaccionismo simbólico, fundamentada en la acción social, la cual, es elaborada por un individuo que opera a través de un proceso en el que advierte, interpreta y valora las cosas, construyendo un plan de acción proyectado en los demás actores sociales. Esto significa que para abordar y analizar la acción social hay que observar a los demás para así reconocer las experiencias y conocimientos de los mismos. Por ello se hace necesario que el individuo reconozca su acción, en cuanto estudie la perspectiva de interacción, atendiendo al modo en que se ejecuta, y no recurriendo a condiciones precedentes como causas explicativas.

En efecto la compleja concatenación de los actores que configuran las organizaciones, instituciones, división del trabajo y redes de interacción, no constituye algo estático, sino dinámico, ya que los seres humanos se complementan en la acción social.
Partiendo de estas proposiciones, es necesario que las organizaciones humanicen los modelos gerenciales, por lo cual, es primordial, que cada gerente, además de conocerse, sea suficientemente responsable para evaluar sus actos, sin desligarse de su equipo de trabajo, en una perspectiva positiva de perfeccionamiento, a fin de darle el significado óptimo a la autovaloración y conseguir la autorregulación, a través del desarrollo y el crecimiento personal, de tal manera que sea capaz de profundizar $y$ clarificar cada vez más su cosmovisión y filosofía de trabajo.

En síntesis, el cambio emplazado por el Estado venezolano a la comunidad universitaria, pretende horizontalizar la respuesta, no solo en el diagnóstico, sino en lo consultivo para hallar soluciones $y$ abordajes participativos que incorporen a todos; no solo en dígito sino en su configuración, en la práctica, consintiendo una gerencia que incluya a sus actores en las decisiones y asigne responsabilidades; que abra puertas y no que las cierre. Una gerencia académica - administrativa que posibilite la pluralidad, pero no por egolatría académica o por rebuscamiento; sino sobre todo por eficacia, exploración y encuentro de la excelencia de la mano de los que en ella hacen vida.

\section{REFERENCIAS}

Blumer, H. (1969). El Interaccionismo simbólico, perspectiva y método. Universidad de California

Clemenza, C., y ferrer, J. (1999). UniversidadEstado-Sector Productivo. Revista de Ciencias Sociales. Volumen №3. Diciembre.

Constitución de la República Bolivariana de Venezuela (1999). Gaceta Oficial de la República Bolivariana de Venezuela.5453 (Extraordinario), Marzo 24, 2000. 
Denzin, N.K. (1970). Sociological methods. A sourcebook. Chicago, IL: Aldine Publishing Company.

Dichter, E. (1988). ¿Es usted un buen gerente? México, México: McGraw Hill.

Frondizi, R. (2001). ¿Qué son los valores? México D.F., México: Breviarios del Fondo de Cultura Económica.

González, G. y Hernández, T. (2000). Análisis e interpretación de la información en la investigación cualitativa. Barquisimeto, Venezuela: Universidad Pedagógica Experimental Libertador.

Guendel, Ludwig. (2001). Políticas públicas y derechos humanos. Observatorio ciudadano, UNICEF.

Guijarro y Chávez (2006). Ética y gerencia universitaria. En Revista Venezolana de Gerencia.

Instituto de Estudios Superiores de Administración (IESA) (1999). ¿Gerentes $y$ negociadores? 5 (2)

Kliksberg, B (1995). La modernización del estado para el desarrollo social. Conferencia dictada en seminario realizado en Bogotá.

Kliksberg, B (1997). Hacia una gerencia social eficiente, algunas cuestiones claves. En: Políticas Públicas y gestión social. Bogotá, Colombia: Alcaldía de Medellín.

Krygier, A. (1988). Consultores de Gerencia: ¿Terapeutas de las organizaciones? Gerente.

Ley Orgánica de Educación (2009). Gaceta Oficial $N^{\circ} 5.929$ (Extraordinario)

. Caracas: 15 de agosto de 2009.

Licha, I., (1999). El enfoque de gerencia social. Banco Interamericano de Desarrollo. Washington, Estados Unidos:
BID, INDES, Recuperado de [http://decon.edu.uy/100jovenes/materi ales/sgNC-14.pdf].

Malpica. R. y Rossell, R. (2010). Gerencia universitaria tiempo de reflexión. .Documento en línea disponible en. [https://www.servicio.bc.uc.edu.ve/educ acion/revista/n35/art10.pdf].

Miquilena Pifia, L. (1999). Problematiea de la formacién docente universitaria. Caracas: Editorial Buchivacoa

Monagas, D. (2003). Consideraciones sobre la gerencia universitaria, en la Escuela de Economía de la Facultad de Ciencias Económicas y Sociales de la Universidad de los Andes.

Muro, X (2003). La Gerencia Universitaria: estudio desde una perspectiva crítica. UPEL. Caracas, Venezuela.

Rojas, G. (2008). Gerencia social. En línea disponible en [http://www.redunirse.net/files/Reflexin es_de_Gerencia_Social.pdf.]

Schütz, A (1995). El problema de la realidad social. Traduc. Néstor Míguez. Compilador Maurice Natason. Buenos Aires, Argentina: Amorrortu, [Links]

Sisk, H. y Sverdlik, M. (1976). Administración y gerencia de empresas. Cincinnati, Ohio, USA: South Western Publishing Co.

Taylor, F. (1911). Principios de la administración científica. México, México: Herrero Hnos.

Universidad de Los Andes (2001). Papeles para el cambio. Informes de las mesas de trabajo (Mesa 0 a Mesa 3). Talleres Gráficos, Mérida, Venezuela: Universidad de los Andes. 